\title{
Assessing the unidimensionality of political opinions. An indirect test of the persuasion bias.* $^{*}$
}

\author{
Lionel Page \\ Queensland University of Technology
}

\begin{abstract}
In an influential paper, DeMarzo, Vayanos, and Zwiebel propose a modelf of persuasion bias whereby people are overly influenced by repetitive information. Such a persuasion bias leads political opinions to be unidimensional with individuals converging to a single "left-right" dimension on every issues. Using a large dataset on political opinions on a wide range of issues just before a presidential election in France, I test whether political opinions are indeed unidimensional. I find that political opinions are far from being unidimensional and I discuss what it means for the persuasion model.
\end{abstract}

Keywords : social preferences, voting behavior, online survey

JEL Classification : A13,D72

Electing officials in modern democracies requires to chose a program covering a wide range of diverse issues involving, among others, economic policy, cultural norms, geopolitical strategy and environmental choices. In spite of the complexity of these issues, the political debate seems most often structured around a single left-right dimension. This dimension typically opposes parties favourable to redistribution (left) to parties aiming to protect property rights and economic freedom (right). In an influential paper, DeMarzo, Vayanos, and Zwiebel (2003), hereafter DVZ, proposed an explanation for this apparent one dimensionality: a persuasion bias. They consider the situation where voters who receive signals from other voters over repeated periods would fail

\footnotetext{
*Lionel Page, School of Economics and Finance, Queensland University of Technology, 2 George street, 4000 Qld, Australia. email: lionel.page@qut.edu.au.
} 
to discount repetitive information. In such a case, they show that in the long term political opinions would tend to line up along a single political dimension.

In the present paper we investigate the dimensionality of the political space. We use a large dataset of answers to an online survey during the French 2007 presidential election. It asked respondents to answer to a wide range of 30 questions relative to the French political debate. Questions were chosen to differentiate as a much as possible the different positions of the different candidates on different issues both in terms of economic policies and societal choices (eg. adoption by gay couples). We use the answers to this questionaire to ascertain whether or not political opinions are onedimensional. France presents an ideal test for DVZ theory. Since 1958, its political system has been mainly stable with political elections opposing two main moderate right and left parties. This time scale should have given enough time to political opinions to converge along a single dimension.

Overall we find, unsurprisingly, that one left-right dimension indeed dominates the opposition of political opinions. However, political opinions are far from being unidimensional. Looking at different measures of dimensionality, it appears that many dimensions (in practice between 3 and 6) are relevant to adequately reflect the complexity of the political space in the 2007 French presidential debate. We suggest that this result is not so much a contradiction of the idea of a persuasion bias than of the idea of a stable political landscape which is assumed by (DeMarzo, Vayanos, and Zwiebel 2003).

This study contributes to the literature on the formation of political opinions. While the unidimensionality of the political space is commonly assumed, this hypothesis is most often not tested. It has been suggested theoretically that a two dimensional space may be better able to represent the position of political opinions on economic and non economic issues (Roemer 1998). A two dimensional space has also been proposed in political science as as better choice to represent political opinions (Kitschelt 1994). In the present study, we use a range of statistical approaches to investigate whether a unidimensional space is able to capture most of the variations in political opinions in the 2007 French presidential election.

The remainder of the article is as follow: Section 1 presents the data, and Section 2 the estimation strategy and the results. Section 3 discuss the results and concludes. 


\section{The data}

\subsection{Data collection}

We use here the data from a large online survey during the French presidential campaign in Spring 2007. We devised a questionnaire which included 30 questions about the major issues of the French public debate $!^{1}$ The questions were determined in collaboration with political journalists from French radio station RTL (first in national audience at the time). They were selected in order to discriminate as much as possible between the different positions of the candidates. This corresponding survey was presented as a quiz and was an adaptation to the French elections of websites like VoteMatch (USA) VoteMatch (NL) or On the Issues (USA), The goal of these websites is to facilitate voters' decisions by giving them information on the candidates closest to their views on a range of political issues. The candidates themselves answered the survey at the end of February.

This survey, named "Polimetre" was available online between the 10th March and the 22th April 2007 (the day of the first stage of the election). People could answer the questions on a Likert scale from 1 to 4 from "strongly agree" to "strongly disagree", with the option to opt for a "no opinion/don't know" position. At the end of the quiz they got in return a ranking of the candidates according to their political distance from them (from the closest to the furthest). Overall, the quiz received 44,082 answers with non missing values ${ }^{2}$ Figure 1 represents a typical feedback screen respondents received after answering the survey. The feedback screen was listing the different candidates by order of proximity as a function of the differences between the respondent and the candidates' answers.

In addition to the 30 opinion questions, a series of other relevant questions were also asked. First we recorded a range of socio-demographic variables (age, gender, level of education, income) and some additional control variables like the level of interest in politics and the candidate they voted for in the last election. Second, we asked respondents their preferred candidate, the candidate they intended to vote for at the election ${ }^{3}$ and their opinion about the skills and personality of the candidates.

\footnotetext{
${ }^{1}$ This questionaire was jointly designed by the author, Paul-Antoine Chevalier and Nicolas Baumard.

${ }^{2}$ A typical phenomenon with such online quizzes is the occurrence of multiple responses from a single respondents. To limit as much as possible the number of multiple responses, our retained sample only contains the first answer submitted for a given ip address (and it ignores later answers submitted from this ip).

${ }^{3}$ These answers can be different due to strategic voting.
} 


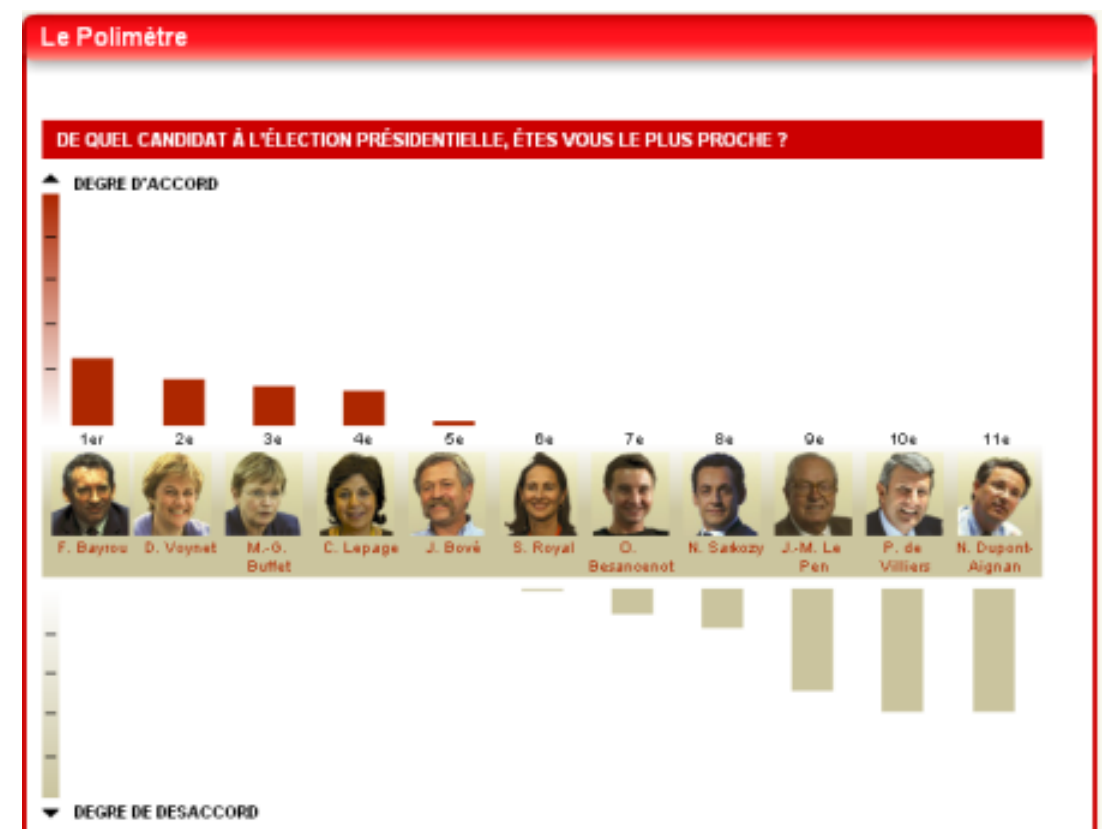

Figure 1: Result window for the online quiz. The window's title translates "From which presidential candidate are you the closest?" The different candidates are presented in order as a function of the distance between their answers and the respondent's answers. 
To measure the political distance we asked each candidate to answer our questionnaire. With the exception of one candidate on the far left (marginal in terms of vote), all candidates answered the questionnaire. For each question we matched the answers of the participants on the 30 political questions and the answers of the candidates 4

\subsection{Sample characteristics}

One important issue concerning our sampling method is the non random selection of respondents. The respondents self-selected themselves in the survey. Therefore the sample is likely to have an over-representation of people both interested in politics and using frequently the Internet. Another important element influencing the sample selection is the choice of websites where the quiz was advertised. We worked with two websites, RTL2007 and Debat2007. The former was the special elections website of the biggest French radio station and the latter was the special elections website of the French think tank, Institut de l'Entreprise (Corporate Institute). RTL is a mass media and is not supposed to be elitist or politically biased. Debat2007 is more elitist and its audience may be right-wing leaning.

A good way to study the specific characteristics of our sample is to compare the distribution of the socio-demographic variables in it to Census data and data from national representative surveys. ${ }^{5}$ Our sample is characterized by a larger proportion of males $(66 \%)$ which is likely to be due to the highest percentage of males using the Internet at that time (Bimber 2000, Ono and Zavodny 2003, Frydel 2006) and also maybe the gender differences in interest in political life (CEVIPOF, 2007). Our sample is quite young. The average age is 34 year old and the median age is 31. A likely explanation is that young people were in 2007 more frequent users of the Internet than older people (Frydel 2006).

We also checked how the positions of the respondents in the sample compared relative to the previous vote at the presidential election in 2002. We observe an over representation of votes for Jospin the mainstream socialist candidate. This could be due to the age distribution of our sample (younger people voting more often for the left), or to a recall bias in respondents' answers ${ }^{6}$. Regarding the distribution of vote intentions in 2007, our respon-

\footnotetext{
4 The two smallest candidates in the race were not included in the result window for the respondents. These two candidates received $1.15 \%$ and $0.34 \%$ of the share of the votes in the elections. For this reason, the window in Figure 1 only displays 11 candidates.

${ }^{5}$ Detailed tables are in appendix.

${ }^{6}$ Lionel Jospin was not qualified to the second stage of the election due to a dispersion of his electorate on smaller candidates. A lot of left wing voters regretted their first stage
} 
dents are more likely to vote for Bayrou, a centrist candidate who arrived third in the 2007 elections.

Overall our sample is therefore not representative of the general population. However to test the unidimensionality of the political space we do not need for the respondents to be distributed in the political space in a representative way. So the characteristics of our sample are not an issue for our empirical investigation. Intuitively, from the point of view of the persuasion bias, a population of respondents interested in politics is likely to have been recipients and senders of more signals about their views. If anything it is in such a population that the unidimensionality should be the clearest. Respondents who are less interested in politics may be more likely to have less consistent political views which do not align on a single dimension. In that regard our sample of respondents is a good setting to test the prediction that political opinions should tend to become unidimensional as people discuss and exchange about their political views.

\subsection{Method}

We assess the unidimensionality of political opinions using several statistical approaches.

First, we use traditional diagnostic tools used in psychology to assess whether answers to a series of questions are reflective of an underlying single dimension. The most common of these tools is the Cronbach's alpha, used to ascertain whether a series of questions may be considered as measuring a single trait. In our context, the Cronbach alpha can help us asess whether there is a single left-right political dimension which is captured by all our questions. We also use a principal component analysis 7 The principal component analysis helps us assess what is the part of the variance in the answers which can be explained by one or more dimensions.

Second, we compute DVZ's index of disagreement on each belief dimension $l$ for agent $i$ :

$$
d_{i, l}=\frac{x_{i, l}-\bar{x}_{l}}{\frac{1}{N} \sum_{j}\left\|x_{j}-\bar{x}\right\|}
$$

DVZ found that a persuasion bias defined as agents taking any signals from others in their network as entirely new information will lead to political opinions converging to a unique position and, in the process, they will get more

choice afterward and they could be reluctant to declare that they did not vote for him.

${ }^{7}$ Note that as our answers are on a Likert scale, we make the assumption that values on the scale provide us with a linear approximation of attitudes. 
unidimensional using the above index of agreement. Agents who are on average on one side of the left-right spectrum relative to others will tend to be on the same side for all questions. To investigate whether this pattern is indeed observed, we measured the average relative position of each respondents:

$$
\bar{d}_{i}=\frac{1}{30} \sum_{l} \frac{x_{i, l}-\bar{x}_{l}}{\frac{1}{N} \sum_{j}\left\|x_{j}-\bar{x}\right\|}
$$

To give the best chance to the unidimensionality hypothesis, we recoded each question in what seemed to us a left-right dimension. Specifically, we reviewed the list of questions. In the design of the survey, we purposely had not all Likert questions coded from left to right. Nine questions appear to be coded in a reverse direction as the others: 1, 2, 8, 10, 11, 14, 16, 23, 26. We therefore reversed them to have all questions coded in the same left-right dimension. We then used the results from the Cronbach's alpha measure to fine tune this coding choice (see below).

We then measured an index of consistency on each issue. To do so we recoded all the distances $d_{i, l}$ on each issues as positive if they were in the same direction of the average individual position $\bar{d}_{i}$, and negative otherwise. It allows us to observe very quickly the proportion of answers on specific issues which are on the other side of the political spectrum relative to the respondent average position. Specifically we measured:

$$
c_{i, l}=\left(d_{i, l}-\bar{d}_{i}\right)(-1)^{\mathbb{1}_{\left\{\bar{d}_{i}<0\right\}}}
$$

\section{Results}

The Cronbach's alpha of our 30 political questions is 0.787 . In psychology, a Cronbach's alpha between 0.7 and 0.8 is interpreted as an indication of an acceptable consistency of the questions and a Cronbach's alpha of 0.8-0.9 as an indication of a good consistency. The Cronbach's alpha is high when the different questions code for a one dimensional trait (here it would be the position on a left-right scale). However it can also be high in situations where the data is not unidimensional. Further diagnostics are therefore required. Before we carry these diagnostics, we can interpret our 0.787 Cronbach alpha as being compatible with some unidimensionality in the data. Note that the calculation of the Cronbach's alpha also provides an indication of which item may need to be reverse coded to fit the underlying dimension in the data. We found that questions 6,8 and 29 needed to be reverse coded. These add 


\begin{tabular}{lccccc}
\hline Component & Eigenvalue & CI low & CI high & Proportion & Cumulative \\
\hline Component 1 & 5.06539 & 5.001241 & 5.147927 & 0.1688 & 0.1688 \\
Component 2 & 2.51247 & 2.484569 & 2.556221 & 0.0837 & 0.2526 \\
Component 3 & 2.06486 & 2.0389 & 2.095307 & 0.0688 & 0.3214 \\
Component 4 & 1.18641 & 1.174281 & 1.205385 & 0.0395 & 0.361 \\
Component 5 & 1.03088 & 1.021579 & 1.044921 & 0.0344 & 0.3953 \\
Component 6 & 1.01263 & 0.9997488 & 1.026535 & 0.0338 & 0.4291 \\
Component 7 & 0.968337 & 0.9605274 & 0.984258 & 0.0323 & 0.4614 \\
Component 8 & 0.960177 & 0.9466664 & 0.970905 & 0.032 & 0.4934 \\
Component 9 & 0.922621 & 0.9121062 & 0.9357169 & 0.0308 & 0.5241 \\
Component 10 & 0.861673 & 0.8522595 & 0.8737427 & 0.0287 & 0.5528 \\
\hline
\end{tabular}

Table 1: First components of the PCA analysis on the answers to the 30 political issues. The CI at $95 \%$ is computed by bootstrap. The last two columns present the proportion of variance explained by each component.

to the nine questions we reverse coded by hand. On these our judgement was validated as the Cronbach's alpha calculation did not suggest we should reverse them back to their original direction. The three questions we missed are about an environmental issue (GMO), an institutional reform (elections of MPs), and Europe (benefit of the EU).

The result of our PCA add further insights to the Cronbach's alpha measurement. Figure 2 and Table 1 show the result of the PCA. Here again the evidence of unidimensionality is mixed. The largest eigenvalue stands out above others but the first component it represents only explains $16 \%$ of the variance in the data. This first component codes for left-right dimension as evidenced by the fact that the factor loadings are positive for each questions on this component. It therefore captures the same dimension we subjectively identified as left-right and which was confirmed by the Cronbach's alpha (with the exception of 3 variables). The second component seems to primarily capture a patriotic/politically conservative dimension which can be found on both sides of the political spectrum.

Several rules of thumbs exist to consider how to select components. The Kaiser's stopping rule recommends to retain the components with an eigenvalue above 1 . Using this method, we would retain 6 components as 6 eigenvalues are significantly above 18 Our political space is therefore a 6dimensional space. The screeplot test proposes instead to abandon components as soon as eigen values stop dropping sharply. Using this method, we

\footnotetext{
${ }^{8}$ Eigenvalue confidence intervals are very tight. They are estimated by boostrap.
} 

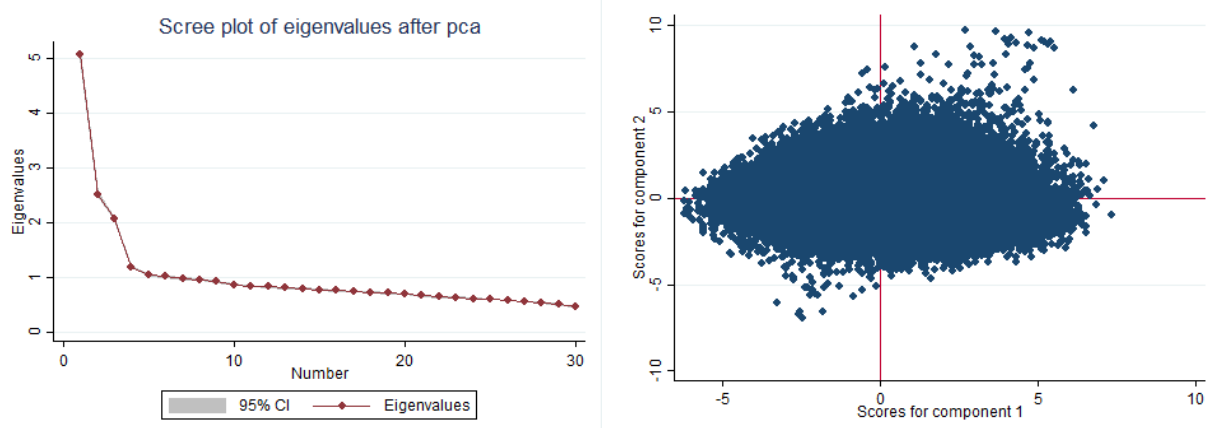

Figure 2: Principal Component Analysis of the answers to the 30 political questions

would retain 3 components totalling $32 \%$ of the total variance in the data.

It therefore appears that while one dimension predominates, the data is not strongly unidimensional. Figure 2 (right panel) shows our respondents answers plotted in the space with the first two components. A first dimension emerges horizontally, but a lot of variance is also explained by the second component.

Using DVZ's index of disagreement, we can further assess the extent to which political opinions are unidimensional. With unidimensional opinions, respondents answers should always be on the same side as the average opinion in the sample: either all on the left or all on the right. In the specific case where the network structure is linear with people only interacting with other people who are directly to their left or right, DVZ's model also predict a polarization of opinions with opinions getting more extreme at both ends over time.

To investigate this Figure 3 plots the average disagreement index (2) over the whole sample of respondents using a flexible non parametric kernel density estimation. The clear bell shaped pattern does not point to any polarization as such, though we observe that the distribution of disagreement has more kurtosis than the normal distribution with the same standard deviation. The observed distribution of disagreement has therefore more observations at the extremes than if the respondents opinions were formed as an average of independent signals. This deviation from normality is significant using both a kurtosis test $(p<0.001)$ and a Shapiro-Wilk test of normality $(p<0.001)$. This kurtosis can be seen as suggesting a step in the direction of polarization.

Figure 3 masks the variability within each respondents. The key prediction of the DVZ model is that agents should become progressively more consistent in their views with the same relative position on a left-right axis 


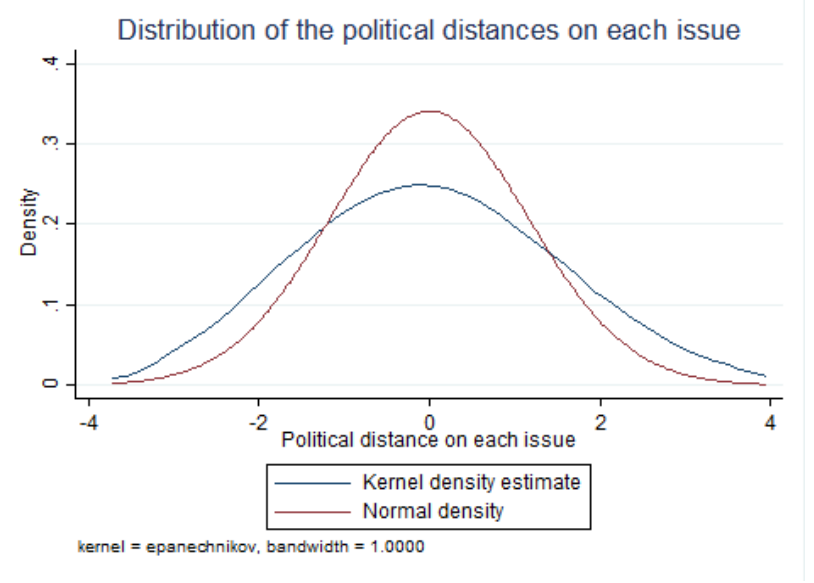

Figure 3: Distribution of disagreements relative the average opinion on each question

on each issue. To investigate our respondent's consistency, we change the direction of all their answers toward the direction of their own average. Hence positive disagreements on a given issue means that on that issue they are on the same side of the average opinion than their average opinion is in general (e.g. they are on the left on this issue and they are on the left on average on all issues). After this change, a negative disagreement on a given question means that the respondent has given an answer in contradiction which his average political position (e.g. on average the respondent is on the left of the answers on all questions but he is on the right for this question).

Figure 4 (left panel) represents the density of the measure of consistency (3) in respondents' answers. Somewhat surprisingly to us, answers to political issues display a lot of inconsistency. On average, respondents take a stance on the other side of their political position in $35 \%$ of their answers. Another pattern we observe is the non normality of the distribution with a much larger kurtosis indicating larger number of large deviations from normality than would be predicted from random deviations around an average position. The deviation from normality is significant both with a kurtosis test $(p<0.001)$ and a Shapiro-Wilk test $(p<0.001)$.

A further investigation shows that this large proportion of "inconsistent" positions on specific issues is not driven by "centrist" respondents being fairly indifferent to left right positions. It is actually widespread across the majority of respondents in the sample. Figure 4 (right panel) shows the distribution of the number of contradictions among respondents. The average number of contradiction is surprisingly high: 10 out of 30 questions. Moreover, it is clear that the previous result is not driven by a subgroup of inconsistent or 

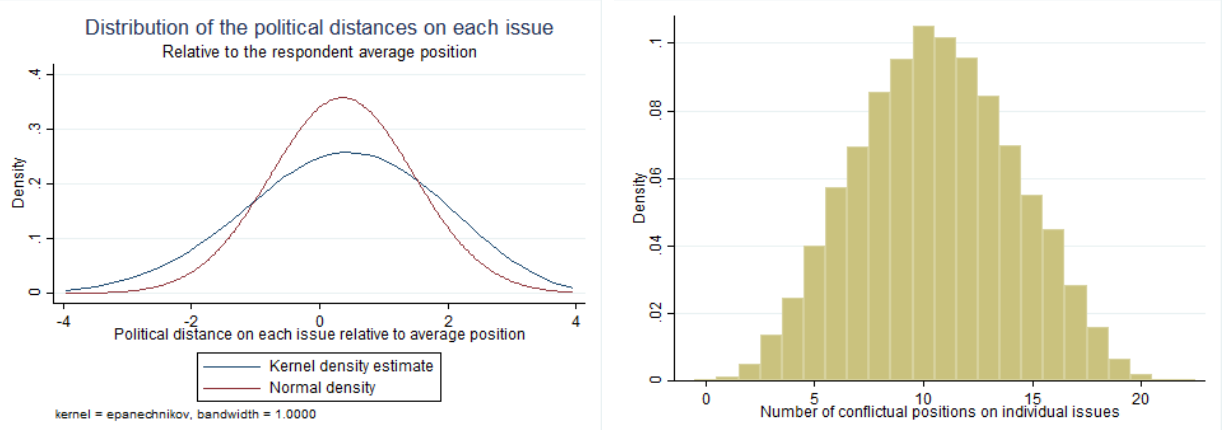

Dispersion of respondent's position

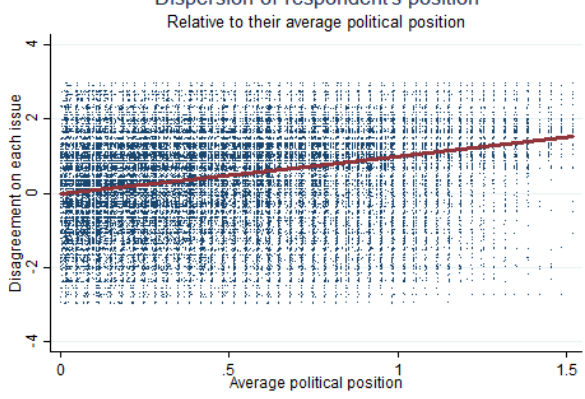

Distribution of the political distances on each issue

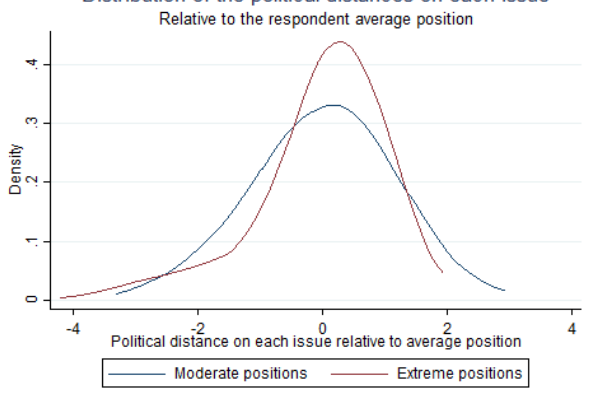

Figure 4: Internal consistency of respondent answers: the left panel represents the proportion of disagreement index falling below 0 indicates the frequency of answers in contradiction with the general political position of the respondent; the right panel represents the distribution of the number of questions where the respondents' answer was in contradiction with his average political position. 
indifferent respondents (e.g. centrists). On the contrary, almost no respondent is consistent. This result is clearly is contradiction with the prediction from DVZ.

\section{Discussion and conclusion}

Overall, our results do not support the idea that the French political space in 2007 was close to being unidimensional. While one left-right dimension emerges, it does not capture the bulk of the variance of answers in our data. Several other dimensions are also present in the data. In contradiction with the unique left-right dimension most often assumed as characterising the political space (or even the 2 dimensional extension sometimes used in political science), our results are suggestive of 3 or more political dimensions. Political opinions appear therefore far from having converged towards a unique political dimension. An interesting aspect of this result is that France, if anything, would a priori seem to be a good candidate for a unidimensional political space of opinion. The left-right divide is very strong in the public discourse with ideological arguments about left-right oppositions often shadowing discussions about competence for elected positions. Moreover, unlike Germany or the UK, the French Socialist Party which dominates the left has never officially abandoned its marxist roots. While it has adopted a social democrat style of government its leaders have been reluctant to openly renounce to marxism not to face the threat of a rising far-left.9 As a consequence, the political discourse is often pitching parties as representing radically different views along a classical left-right axis. It is therefore interesting to note that our respondents' answers were actually only imperfectly reflecting one political dimension.

As an indirect test of the persuasion bias model proposed by DVZ, our results do not provide a lot of support for its prediction. As the model predicts a convergence over time, it may well be that this convergence has not yet taken place or that the past political space was highly non one-dimensional. Unfortunately the French political space has been fairly stable since the start of the 5th Republic (1958) with two leading parties, a moderate conservative and a socialist party dominating the presidential and parliamentary elections 10

\footnotetext{
${ }^{9}$ The communist anthem is still one of the sung in some political rallies. In 2011 several MPs from the Socialist Party, sang this anthem in the National Assembly as a movement of protest towards the conservative government.

${ }^{10}$ These parties have changed names over the period. The main conservative party has been: UNR (1958-1968), UDR (1968-1976), RPR (1972-2002), UMP (2002-2015), Les Republicains (2015-). And the main socialist party has been: SFIO (1905-1968), PS
} 
One may therefore think that there has been enough time for convergence to take place or at least for some signs of convergence to be observable. Our result does not point to a marked unidimensionality which may cast some doubts about this empirical prediction of the persuasion model.

That being said, it may not mean that the intuitions of the model on persuasion bias are flawed. Instead, our result may reflect that the prediction about unidimensionality of policial opinions "in the long run" may not have a lot of empirical relevance in the short run. Can we make sense of this lack of unidimensionality with such a perspective? The non-unidimensionality in our data may actually point out to directions for future models of persuasion. For instance, even if the persuasion model adequately reflects the dynamics of opinion, it does not take into account that voters leave and enter the network as old generation of voters cease to vote and new generation of young voters reach the voting age. Intuitively, if the generations of voters get renewed too fast relative to the speed of convergence, the political space could fail to converge towards a one dimensional space. Another possible departure from the initial persuasion model is that some agents may be pure suppliers of opinion instead of receivers (e.g. news outlets, political groups). If some agents are indeed only providers, they may retain an audience around their initial political position which may not be aligned on one political dimension. For instance a religious group may be left-wing on some issues (reduction of inequalities) and right-wing on other issues (gay mariage). As long as such a group does not change its position as a function of the position of others, it may contribute to keeping the political space multidimensional.

The processus of convergence can also be affected by external changes to the political space. First, new issues can arise which split the political space according to unexpected lines. In France, the issue of European integration could be such a question. While mainstream parties have long supported European integration, both the mainstream rightwing party and the socialist party split in two during the debate of the 2005 referendum on the European Constitution. Some rightwing conservatives called to vote against it to protect national sovereignty, and some leaders of the socialist party as well as the far-left called to vote against it to protect social regulations from "liberal" rule under the proposed text of the Constitution. As such new issues may arise from time to time ${ }^{11}$ they may maintain the political space multidimensionality even if at any moment in time it tends to converge towards a one dimensional political space.

${ }^{11}$ The debate over the female Muslim veil in public space is also an issue which has split the space along lines different from the typical left-right dimension.
} 


\section{References}

Bimber, B. (2000): "Measuring the gender gap on the Internet," Social Science Quarterly, 81(3), 868-876.

DeMarzo, P. M., D. Vayanos, and J. Zwiebel (2003): "Persuasion Bias, Social Influence, and Unidimensional Opinions," The Quarterly journal of economics, 118(3), 909-968.

FRYDEL, Y. (2006): "Internet au quotidien : un Français sur quatre," INSEE Premières.

Kitschelt, H. (1994): The transformation of European social democracy. cambridge university press.

Ono, H., And M. Zavodny (2003): "Gender and the Internet," Social Science Quarterly, 84(1).

Roemer, J. E. (1998): "Why the poor do not expropriate the rich: an old argument in new garb," Journal of Public Economics, 70(3), 399-424. 


\section{A The political questions}

Some questions are hard to translate due to the specificity of the French political debate. The questions marked with a ${ }^{e}$ are in the "economic" list and the one marked with a ${ }^{p}$ in the "political" one.

1. To defend their opinion, some, like the militants against Genetically Modified Food, sometimes infringe the law (eg civil disobedience). What do you think of this kind of action? ${ }^{p}$

2. Homosexual couples should have the right to adopt children. ${ }^{p}$

3. It is necessary to create a compulsory civil service for everyone (NB:civilian equivalent to the military service). ${ }^{p}$

4. Experiments on the human genom as they are currently done are immoral. ${ }^{p}$

5. Minors delinquents should be sent to jail. ${ }^{p}$

6. Genetically Modified Organisms are a danger for humanity and should be strictly forbidden. ${ }^{p}$

7. Lowering immigration would lower unemployment in France.

8. MPs should be elected with a proportional voting system. ${ }^{p}$

9. Housewives should have a "mother income". $e$

10. No obligation of minimum service should be enforced in case of strike in the transport system. ${ }^{e}$

11. There is not enough public firms today. ${ }^{e}$

12. Merit should have a higher role in the determination of civil servants pay. ${ }^{e}$

13. Economically, France is declining.

14. The law on the 35 hour work week law has been a social progress.

15. Unemployed refusing three successive job offers should be sanctioned financially. ${ }^{e}$

16. Trade Union membership should be compulsory. ${ }^{e}$

17. The private part in the financing of pensions should increase. ${ }^{e}$ 
18. The age of retirement should be pushed up. ${ }^{e}$

19. Globally, the skills of kids at a given level of school is decreasing over time.

20. The state geographical regulation over the choice of school should be suppressed. $^{e}$

21. The University fees should be increased. ${ }^{e}$

22. Increasing the proportion of children reaching higher level of education only decreases the value of diplomas.

23. Tax on inheritance are legitimate and should be maintained. ${ }^{e}$

24. In the current context, it is urgent to lower the income tax. ${ }^{e}$

25. The level of public taxation infringe the country's growth. ${ }^{e}$

26. The budget of the Defense Ministry is too high. ${ }^{p}$

27. France should be closer from the USA on an international point of view. ${ }^{p}$

28. Turkey should not enter in the EU. ${ }^{p}$

29. Globally, France benefits from belonging to the EU. ${ }^{p}$

30. A new European Constitution should be proposed to vote. ${ }^{p}$

\section{B Detailed descriptive statistics on the sam- ple}

Table 2 compares the sample with the statistics of the French Institute of National Statistics (INSEE). Table 3 compares the vote in 2002 between the sample and the whole population. Table 4 compares the vote in 2007 between the sample and the whole population. 
Table 2: Socio-demographic characteristics of the sample compared to the French population (using the 2005 Census and the 2007 Bilan Demographique).

\begin{tabular}{lccccc}
\hline Characteristics & N sample & \% sample & \% census & Diff & Source \\
\hline Women & 14,717 & 33.39 & 51.4 & -18.01 & Census 2007 \\
Men & 29,365 & 66.61 & 48.6 & 18.01 & Census 2007 \\
\hline Departement 75 (Paris) & 5,562 & 13.10 & 3.54 & 9.56 & Census 2005 \\
Departement 92 & 2,269 & 5.35 & 2.49 & 2.86 & Census 2005 \\
Departement 69 (Lyon) & 1,734 & 4.09 & 2.72 & 1.37 & Census 2005 \\
\hline Women age 17-24 & 3,717 & 25.26 & 14.77 & 10.49 & Bilan Demo 2007 \\
Women age 25-34 & 4,994 & 33.93 & 15.25 & 18.68 & Bilan Demo 2007 \\
Women age 35-44 & 2,522 & 17.14 & 16.83 & .31 & Bilan Demo 2007 \\
Women age 45-54 & 1,939 & 13.18 & 16.46 & -3.28 & Bilan Demo 2007 \\
Women age 55-64 & 1,204 & 8.18 & 13.92 & -5.74 & Bilan Demo 2007 \\
Women age 65-74 & 265 & 1.80 & 10.17 & -8.37 & Bilan Demo 2007 \\
Women age 75+ & 76 & 0.52 & 12.59 & -12.07 & Bilan Demo 2007 \\
\hline Men age 17-24 & 6,812 & 23.20 & 16.36 & 6.84 & Bilan Demo 2007 \\
Men age 25-34 & 10,107 & 34.42 & 16.73 & 17.69 & Bilan Demo 2007 \\
Men age 35-44 & 5,995 & 20.42 & 17.97 & 2.45 & Bilan Demo 2007 \\
Men age 45-54 & 3,284 & 11.18 & 17.1 & -5.92 & Bilan Demo 2007 \\
Men age 55-64 & 2,366 & 8.06 & 14.62 & -6.56 & Bilan Demo 2007 \\
Men age 65-74 & 643 & 2.19 & 9.42 & -7.23 & Bilan Demo 2007 \\
Men age 75+ & 158 & 0.54 & 7.81 & -7.27 & Bilan Demo 2007 \\
\hline Men divorced & 1,092 & 3.74 & 6.9 & -3.16 & Bilan Demo 2007 \\
Women divorced & 942 & 6.44 & 8.4 & -1.96 & Bilan Demo 2007 \\
Men married & 12,127 & 41.5 & 50.5 & -9 & Bilan Demo 2007 \\
Women married & 5,575 & 38.09 & 46.3 & -8.21 & Bilan Demo 2007 \\
Men single & 15,860 & 54.28 & 39.9 & 14.38 & Bilan Demo 2007 \\
Women single & 7,890 & 53.91 & 32.8 & 21.11 & Bilan Demo 2007 \\
Men widow & 142 & 0.49 & 2.7 & -2.21 & Bilan Demo 2007 \\
Women widow & 229 & 1.56 & 12.5 & -10.94 & Bilan Demo 2007 \\
\hline
\end{tabular}


Table 3: Comparison of the respondents' declared vote in 2002 to the actual shares of votes nationally.

\begin{tabular}{lcccc}
\hline Name & N sample & \% sample & \% 2002 & Diff \\
\hline Abstention & 5,027 & 12.52 & 28.4 & -15.88 \\
Informal vote & 1,360 & 3.39 & 2.42 & 0.97 \\
Bayrou (centre right) & 3,736 & 9.31 & 4.73 & 4.58 \\
Besancenot (far left) & 2,237 & 5.57 & 2.94 & 2.63 \\
Boutin (right) & 205 & 0.51 & 0.82 & -0.31 \\
Chevenement (left) & 1,394 & 3.47 & 3.69 & -0.22 \\
Chirac (right) & 8,068 & 20.1 & 13.75 & 6.35 \\
Gluckstein (far left) & 80 & 0.2 & 0.32 & -0.12 \\
Hue (far left) & 667 & 1.66 & 2.33 & -0.67 \\
Jospin (left) & 8,350 & 20.8 & 11.19 & 9.61 \\
Laguiller (far left) & 706 & 1.76 & 3.96 & -2.2 \\
Le Pen (far right) & 2,729 & 6.8 & 11.66 & -4.86 \\
Lepage (centre right) & 554 & 1.38 & 1.3 & -0.08 \\
Madelin (right) & 970 & 2.42 & 2.7 & -0.28 \\
Mamere (left) & 2,737 & 6.82 & 3.63 & 3.19 \\
Megret (far right) & 62 & 0.15 & 1.62 & -1.47 \\
Saint Josse (right) & 192 & 0.48 & 2.92 & -2.44 \\
Taubira (left) & 1,065 & 2.65 & 1.6 & 1.05 \\
\hline
\end{tabular}

Table 4: Comparison of the respondents' declared intended vote in 2007 to the actual shares of votes nationally.

\begin{tabular}{lcccc}
\hline Name & N sample & \% sample & \% 2007 & Diff \\
\hline Bayrou (centre right) & 13,338 & 30.26 & 18.57 & 11.69 \\
Besancenot (far left) & 1,613 & 3.66 & 4.08 & -0.42 \\
Bove (left) & 694 & 1.57 & 1.32 & 0.25 \\
Buffet (far left) & 658 & 1.49 & 1.93 & -0.44 \\
Devilliers (right) & 514 & 1.17 & 2.23 & -1.06 \\
Laguiller (far left) & 224 & 0.51 & 1.33 & -0.82 \\
Le Pen (far right) & 1,804 & 4.09 & 10.44 & -6.35 \\
Nihous (right) & 182 & 0.41 & 1.14 & -0.73 \\
Royal (left) & 11,743 & 26.64 & 25.87 & 0.77 \\
Sarkozy (right) & 11,762 & 26.68 & 31.18 & -4.50 \\
Schivardi (far left) & 63 & 0.14 & 0.33 & -0.19 \\
Voynet (left) & 836 & 1.90 & 1.57 & 0.33 \\
\hline
\end{tabular}

